\title{
Trend of Soil-Transmitted Helminths in Ethiopian Children: A Systematic Review and Meta-Analysis (2000-2018)
}

\author{
Getaneh Alemu $\mathbb{D}^{1},{ }^{1}$ Feleke Mekonnen, ${ }^{1}$ Mezgebu Nega, ${ }^{2}$ and Chalachew Muluneh ${ }^{2}$ \\ ${ }^{1}$ Department of Medical Laboratory Science, Bahir Dar University, Bahir Dar, Ethiopia \\ ${ }^{2}$ Amhara National Regional State Health Bureau, Bahir Dar, Ethiopia \\ Correspondence should be addressed to Getaneh Alemu; getanehmlt@gmail.com
}

Received 7 July 2021; Accepted 23 September 2021; Published 22 October 2021

Academic Editor: Lizandra Guidi Magalhães

Copyright (c) 2021 Getaneh Alemu et al. This is an open access article distributed under the Creative Commons Attribution License, which permits unrestricted use, distribution, and reproduction in any medium, provided the original work is properly cited.

\begin{abstract}
Background. Ethiopia is one of the tropical countries with a heavy burden of soil-transmitted helminths. As a result, the nation has been implementing mass drug administration, water, sanitation, and hygiene and health extension programs to control those parasites. Hence, updated data about the prevalence and trend of parasites over time has a pivotal role to assess the success of existing control programs. Methods. Studies conducted between 2000 and 2018 were searched from PubMed, Google Scholar, and local journals for systematic reviews and meta-analysis following the PRISMA guideline and checklists. Eligible studies were selected based on preset inclusion and exclusion criteria. The quality of the included studies was assessed using the Newcastle-Ottawa Scale in meta-analysis. Heterogeneity between studies was assessed using the Cochran $Q$ test and $I^{2}$ test statistics based on the random effect model. Comprehensive meta-analysis (CMA 2.0) was used to calculate the pooled prevalence, and metaregression was run to assess the trend of parasite prevalence over time. Results. Thirty-eight studies recruiting 16,266 participants were included in the review. The pooled prevalence of intestinal parasites was 52.0\% (95\% CI: 44.4-59.5). Amhara region was with the highest prevalence (60.3\%; 95\% CI: 50.1-69.6). Among soil-transmitted helminths, Ascaris lumbricoides (11.2\%; 95\% CI: 8.4-14.8) was with the highest pooled prevalence followed by hookworms (10.4\%; $95 \%$ CI: 7.9-13.7) and Trichuris trichiura (3.6\%; 95\% CI: 2.4-5.4). Metaregression analysis revealed that all soil-transmitted helminths did not show a significantly decreasing trend over time $(p>0.05)$. Conclusion. Despite various control efforts having been made, soil-transmitted helminths are of high distribution, and their prevalence is not significantly decreasing in Ethiopia. Hence, other control approaches like community-led sanitation should be integrated with mass drug administration to achieve the national goal of soil-transmitted helminth elimination by 2025 .
\end{abstract}

\section{Background}

Intestinal parasitic infections (IPIs) are among the major public health problems worldwide. Globally, 3.5 billion people are infected, among which 450 million manifest illnesses because of the infection [1]. Poor sanitary conditions and conducive climate for the survival of parasites outside the host make the majority of intestinal parasites (IPs) abundant in the tropics [2]. Globally, Ascaris lumbricoides (A. lumbricoides), Trichuris trichiura (T. trichiura), and hookworms infect 819, 465, and 439 million people, respectively. They are grouped under soil-transmitted helminths (STHs) because their infective stages embryonate or develop in the soil [3].
In Ethiopia, 81 million people are at risk of STH infection. School-age children (SAC) account for nearly onethird (25.3 million) of the risk population [4]. Transmission of STHs is associated with personal hygiene and sanitation practices as well as access to safe water. However, the Ethiopian Demography and Health Survey report shows that $40 \%$ and $33 \%$ of households had no hand washing facilities and toilets at their home. Access to safe water for drinking was also poor in the rural community with only $57 \%$ coverage [5]. These conditions contribute to ease of transmission for IPs in general and STHs in particular [6].

Even if all population groups can be infected by STHs, SAC are the most commonly affected groups. This is because 


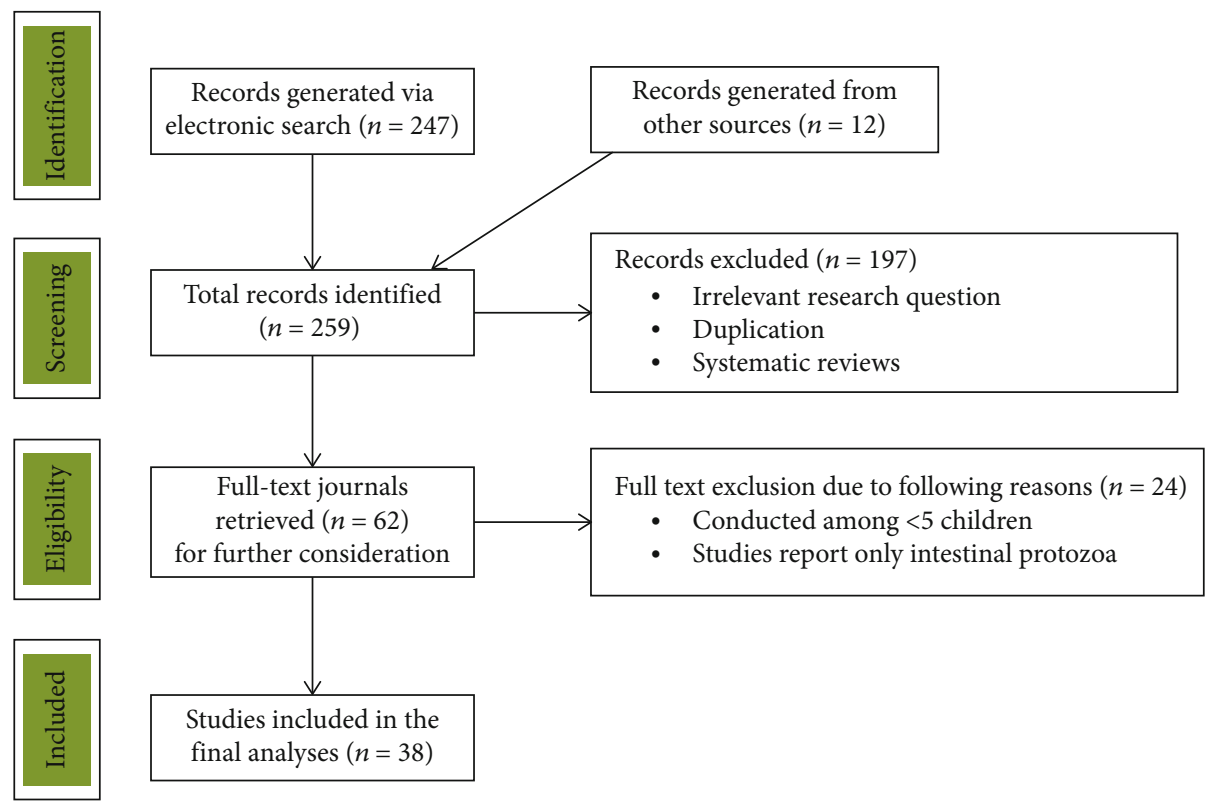

FIGURE 1: Flow chart showing selection process of eligible studies.

of their immature immune system and frequent exposure to infective sources (soil and water) as well as poor hygiene and sanitary practices $[7,8]$. Moreover, children are more vulnerable to serious complications such as prolonged diarrhea, malnutrition, anemia, bowel obstruction, and learning disabilities compared to adults [9]. Hence, the Federal Ministry of Health of Ethiopia has been taking strong measures targeted for the control of IPs among SAC. Implementation of the health extension program since 2003/4, which focuses on the preventive health packages, has brought tangible improvements in the health of the rural community. Creating awareness about latrine construction and utilization as well as keeping personal and environmental hygiene among the community has been one of the priority concerns of the program [10]. Implementation of biannual mass drug administration (MDA) with albendazole or mebendazole for the control of STH since 2015, primarily targeting SAC, has been the other important commitment of the ministry [11].

All these efforts are expected to reduce the burden and health impacts of STHs from time to time. Several survey studies have been conducted among SAC at different geographical settings of the country. However, there is no adequate data reporting the pooled prevalence and trend in magnitude of STHs over time. Hence, we conducted a systematic review and meta-analysis aimed at assessing the pooled prevalence and trend of STH infections among SAC in Ethiopia. The review provides evidence about the impact of ongoing control and prevention programs and to plan for the future. In this review, we did not include Strongyloides stercoralis because we need to focus on the main STHs which have been targeted for MDA-based intervention so that we can analyze the impact of MDA on their prevalence. Moreover, there have been controversies whether Strongyloides stercoralis fulfills the definition of STHs.

\section{Methods}

2.1. Setting. We included studies conducted all over Ethiopia. Ethiopia is located in the horn of Africa at geographical coordinates of $8^{\circ} \mathrm{N}$ and $38^{\circ} \mathrm{E}$ [12]. The country is composed of nine regional states and two city administration councils. Altitude of the country ranges from high peaks of 4,620 meters above sea level to a low depression of 148 meters below sea level. More than half of the country lies above 1,500 meters $[4,12]$. The estimated population of the country for 2018 was 107.53 million where SAC of age 5-14 years account for $31.2 \%[4,13]$.

2.2. Eligibility Criteria. School- or community-based studies conducted in Ethiopia between January 2000 and December 2018 targeting SAC (4-25 years old) and published in the English language were included in the present review. Studies reporting both intestinal protozoa and helminths or intestinal helminths alone were included since our primary targets were STHs.

2.3. Information Sources and Search of Literature. Potential articles were searched in PubMed, Google Scholar, and local journals following the PRISMA guideline and checklists [14]. Search in PubMed was done using the MeSH terms "helminth OR intestinal helminth OR helminthiasis OR intestinal parasite OR parasite OR parasitosis OR intestinal parasitosis OR parasitic infection AND Ethiopia." A manual search was also conducted on all relevant references listed within the articles identified after an initial electronic search. The search was independently done by two reviewers to minimize bias and missing of studies.

2.4. Study Selection. The study selection process is shown in Figure 1. Studies conducted in health facilities were excluded because we expect inflated data as only symptomatic children with parasitic illness participate in healthcare facility- 
TABLE 1: Characteristics of included studies $(n=38)$.

\begin{tabular}{|c|c|c|c|c|c|c|c|c|}
\hline Study name & Study region & Study period & Sample size & Total positive & IP prevalence & Lab method & IP detected & Age group \\
\hline Merem, 2017 [19] & Amhara & 2013-14 & 408 & 282 & 69.1 & FEC & Helminth & $6-20$ \\
\hline Abebe, 2011 [20] & Amhara & 2009 & 319 & 263 & 82.4 & KATO & Helminth & $5-19$ \\
\hline Abebe, 2017 [41] & AA & 2017 & 422 & 100 & 23.7 & DWM, FEC & Both & $4-18$ \\
\hline Ashenafi, 2014 [42] & SNNPR & 2012 & 400 & 324 & 81.0 & DWM, FEC & Both & $5-15$ \\
\hline Abraham, 2013 [21] & Amhara & 2011 & 403 & 235 & 58.3 & KATO & Helminth & $5-15$ \\
\hline Alamneh, 2014 [22] & Amhara & 2011-12 & 385 & 170 & 44.2 & FEC & Helminth & $6-18$ \\
\hline Alemeshet, 2011 [16] & Oromia & 2008 & 855 & 406 & 47.5 & FEC & Helminth & $7-16$ \\
\hline Ayalew, 2015 [43] & SNNPR & 2013 & 460 & 352 & 76.5 & KATO & Both & $5-17$ \\
\hline Bamlaku, 2017 [23] & SNNPR & 2015 & 374 & 254 & 67.9 & FEC & Helminth & $5-15$ \\
\hline Bayeh, 2013 [24] & Amhara & 2011 & 778 & 401 & 51.5 & KATO & Helminth & $7-14$ \\
\hline Begna, 2016 [44] & Oromia & 2013 & 492 & 131 & 26.6 & DWM, FEC & Both & $6-19$ \\
\hline Bereket, 2015 [18] & SNNPR & 2014 & 384 & 328 & 85.4 & KATO, SAF & Helminth & $5-19$ \\
\hline Bereket, 2017 [25] & SNNPR & 2015 & 503 & 363 & 72.2 & KATO, SAF & Helminth & $5-19$ \\
\hline Daniel, 2017 [15] & Amhara & 2017 & 279 & 85 & 30.5 & FEC & Both & $6-16$ \\
\hline Eleni, 2013 [45] & Tigray & 2011-12 & 384 & 233 & 60.7 & DWM & Both & $6-18$ \\
\hline Ephrem, 2015 [26] & Oromia & 2012 & 644 & 89 & 13.8 & McMaster & Helminth & $5-25$ \\
\hline Fikreslasie, 2017 [27] & Oromia & 2014-15 & 321 & 59 & 18.4 & FEC & Helminth & $6-15$ \\
\hline Gemechis, 2013 [28] & Oromia & 2013 & 390 & 182 & 46.7 & KATO, FEC & Helminth & $6-17$ \\
\hline Getachew, 2014 [29] & Amhara & 2013 & 384 & 327 & 85.2 & KATO, SAF & Helminth & $5-19$ \\
\hline Getaneh, 2018 [46] & SNNPR & 2017 & 391 & 181 & 46.3 & FEC & Both & $5-14$ \\
\hline Getaneh, 2019 [47] & SNNPR & 2018 & 351 & 95 & 27.1 & DWM, FEC & Both & $5-14$ \\
\hline Girum, 2005 [30] & Oromia & 2001 & 415 & 113 & 27.2 & FEC & Helminth & $5-24$ \\
\hline Lemlem, 2010 [48] & Tigray & 2007 & 381 & 263 & 69.0 & KATO & Both & $5-19$ \\
\hline Ligabaw, 2014 [31] & Amhara & 2013 & 385 & 372 & 84.9 & FEC, KATO & Helminth & $6-15$ \\
\hline Megbaru, 2014 [32] & SNNPR & 2012 & 405 & 109 & 26.9 & KATO & Helminth & $5-18$ \\
\hline Mengstu, 2014 [33] & Amhara & 2011-12 & 402 & 219 & 54.5 & DWM, FEC & Helminth & 6-19 \\
\hline Mohammed, 2015 [34] & Tigray & 2013 & 442 & 54 & 12.8 & KATO & Helminth & $5-20$ \\
\hline Mulusew, 2014 [49] & Amhara & 2014 & 358 & 245 & 68.4 & DWM, FEC & Both & $7-21$ \\
\hline Nam Linh, 2012 [17] & Amhara & 2008 & 664 & 47 & 7.1 & FEC & Helminth & $6-19$ \\
\hline Serkadis, 2013 [35] & Oromia & 2011-12 & 366 & 233 & 63.7 & McMaster & Helminth & $5-15$ \\
\hline Tadesse, 2018 [50] & Amhara & 2017 & 409 & 193 & 47.2 & Richie's & Both & $7-14$ \\
\hline Tamirat, 2017 [36] & Amhara & $2015-16$ & 359 & 235 & 65.5 & FEC & Helminth & $7-14$ \\
\hline Tamirat, 2018 [51] & Amhara & 2014 & 382 & 200 & 52.4 & FEC & Both & $7-13$ \\
\hline Temam, 2018 [37] & Oromia & 2016 & 340 & 199 & 58.5 & DWM, KATO & Helminth & $6-19$ \\
\hline Teshome, 2019 [38] & Oromia & 2017 & 317 & 224 & 70.7 & KATO, FEC & Helminth & $5-15$ \\
\hline Tilahun, 2015 [39] & Amhara & 2010 & 384 & 211 & 54.9 & KATO & Helminth & $5-14$ \\
\hline Tsega, 2018 [40] & Tigray & 2017 & 410 & 52 & 12.7 & KATO & Helminth & $6-19$ \\
\hline Tsegaw, 2013 [52] & Amhara & 2008 & 520 & 371 & 71.3 & DWM, FEC & Both & $6-15$ \\
\hline
\end{tabular}

AA: Addis Ababa; DWM: direct wet mount; FEC: formol-ether concentration; KATO: Kato-Katz; SNNPR: Southern Nations Nationalities and People's Region.

based studies. Because our interest in this review was the SAC, the most susceptible group for IPI, studies targeting preschool children and adults alone were not considered. Surveys conducted before January 2000 and those reporting only intestinal protozoa were also excluded from the review. Published surveys reporting at least the age group of study participants, location of data collection, and data collection period were included in this review (Figure 1). The search was conducted by two authors separately, and there were differences in the inclusion of the three studies. After discussion, both authors agreed to include two and excluded one study.

2.5. Data Extraction. Two authors extracted data independently using a standard data collection form constructed in Excel. Information was collected about the total number of children that participated, total number of children with laboratory confirmed intestinal parasitosis, age group, year of 


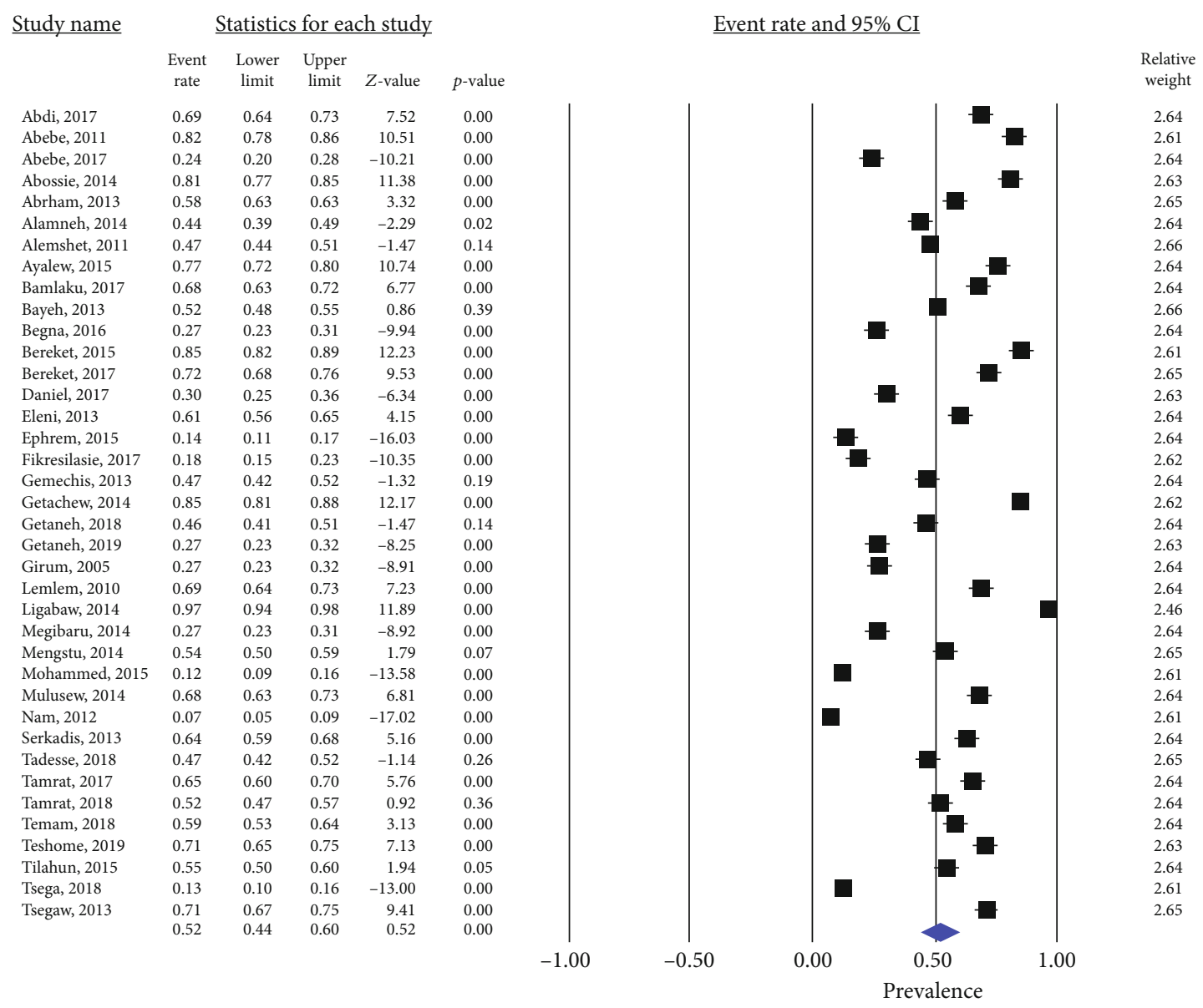

FIGURE 2: Forest plot showing pooled prevalence of intestinal parasites.

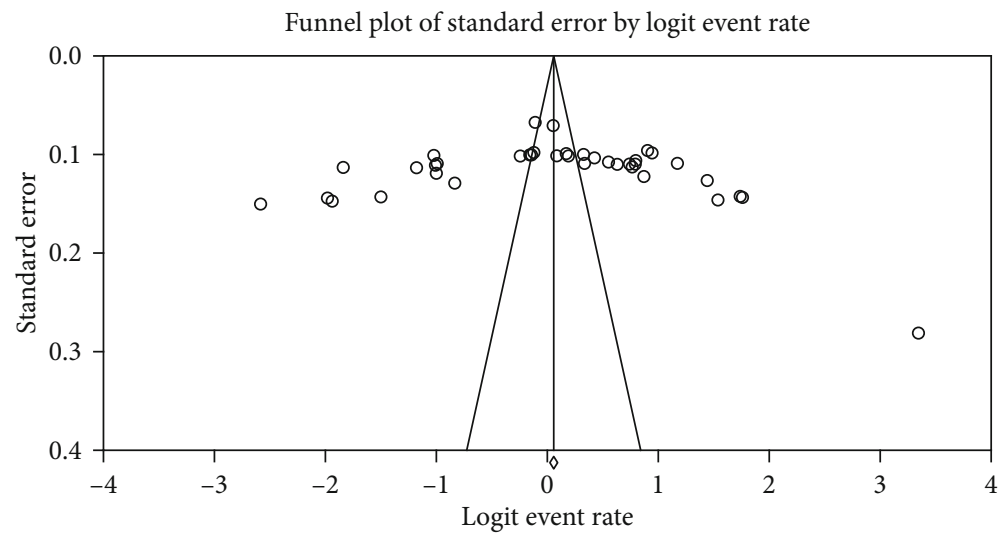

Figure 3: Publication bias assessment funnel plot; Egger's regression test $(p=0.481)$ and Begg's rank correlation $(p=0.470)$.

study, location of sampling (study region), laboratory methods used for investigation of fecal specimens, groups of parasites detected (helminth only/helminth and protozoa), and total number of children infected with the big three STHs (A. lumbricoides, hookworms, and T. trichiura).

2.6. Statistical Analysis. Estimation of the pooled prevalence of IPIs and summary prevalence of each STH was calculated using CMA 2.0 software. Subgroup analysis was done by region of study and study period. The random effect model was used in the analysis because the study populations across selected studies vary, at least in terms of age group and level of risk of IPI. Heterogeneity between studies was checked with a forest plot, Cochran's $Q$ test, and $I^{2}$ test. Significant heterogeneity was declared at $I^{2}>50 \%$ and $Q$ test $(p<0.10)$. Quality of study was checked with the 


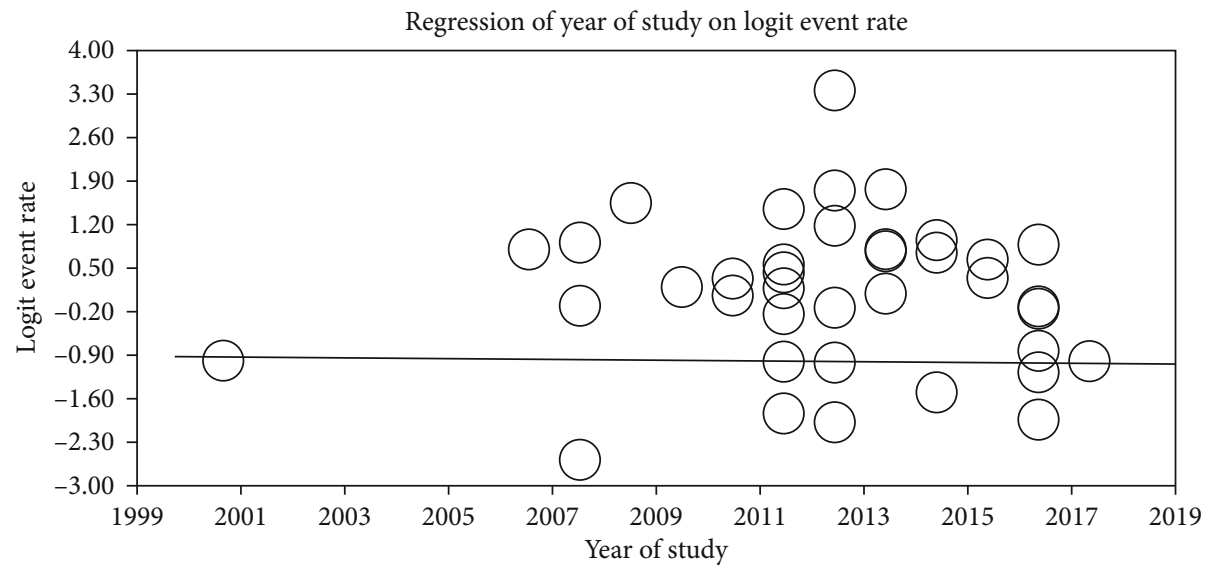

(a)

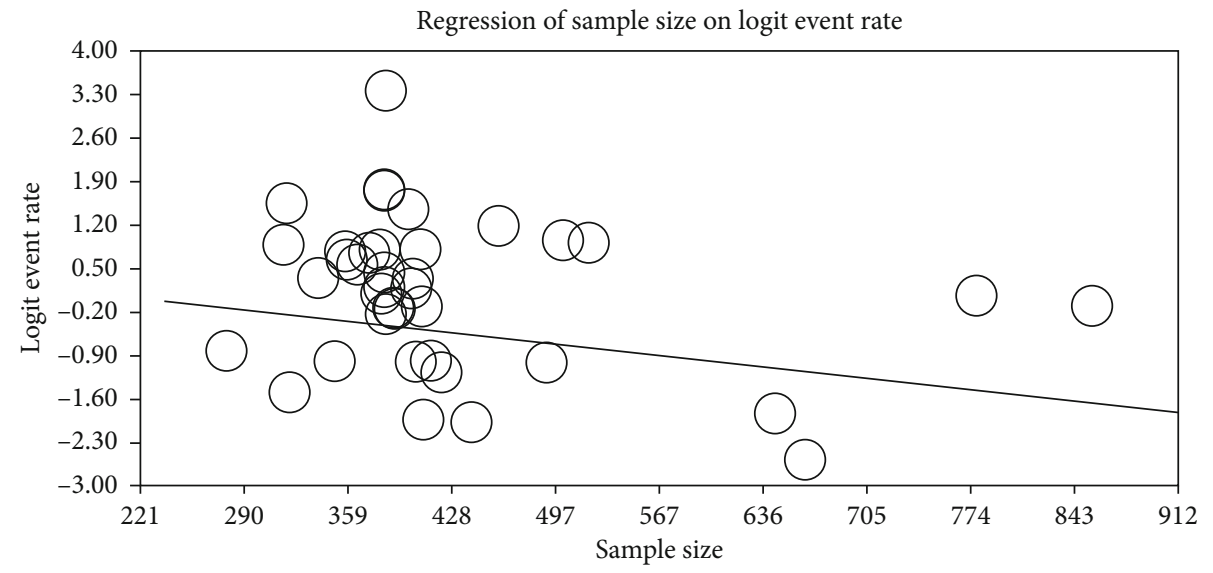

(b)

Figure 4: Metaregression of prevalence of IPs by (a) year of study $(B=-0.00487, p=0.458)$ and $(\mathrm{b})$ sample size $(B=-0.00252, p=0.057)$.

TABLE 2: Metaregression analysis of prevalence of STHs by study period.

\begin{tabular}{lccccc}
\hline \multirow{2}{*}{ Parasite species } & \multirow{2}{*}{ No. of studies } & \multicolumn{2}{c}{ Pooled prevalence \% (95\% CI) } & Overall & $\beta$ \\
\hline Hookworms & 34 & $11.7(8.0-16.8)$ & $8.7(6.5-13.4)$ & $10.4(7.9-13.7)$ & -0.01141 \\
A. lumbricoides & 34 & $12.2(8.2-17.9)$ & $10.0(6.7-14.6)$ & $11.2(8.4-14.8)$ & -0.02173 \\
T. trichiura & 32 & $3.2(1.5-5.5)$ & $4.2(2.2-8.0)$ & $3.6(2.4-5.4)$ & 0.138 \\
\hline
\end{tabular}

Newcastle-Ottawa Scale adapted for cross-sectional study by two reviewers independently, and disagreements were resolved by discussion.

2.7. Risk of Bias across Studies. Presence of publication bias was assessed by drawing funnel plots. Logit event rates of the studies were plotted against standard error so that asymmetry in the distribution of studies can easily be observed. For further assessment of publication bias, Begg's adjusted rank correlation and Egger's regression asymmetry test were also used. Significant publication bias was considered if $p$ $<0.05$ in those tests. Leave-one-out analysis was done to assess the outlier study results responsible for the skewed pooled prevalence of intestinal parasites.

\section{Results}

3.1. Characteristics of Studies. Among the initially identified 259 studies, 38 cross-sectional studies conducted from the years 2000 to 2018 were included in the analysis (Figure 1). A total of 16,266 participants with 4 to 25 years of age range were recruited in the study. The smallest and largest sample sizes among the included studies were 279 and 855 , respectively $[15,16]$. The lowest and the highest IP prevalence among the studies were $7.1 \%$ and $85.4 \%$, respectively $[17,18]$. Nearly half (16 out of 38 ) of the studies were conducted in the Amhara region. Twenty-five studies reported only intestinal helminths [16-40], while the remaining 13 studies reported both intestinal helminths and protozoa $[15,41-52]$. All studies were school-based 


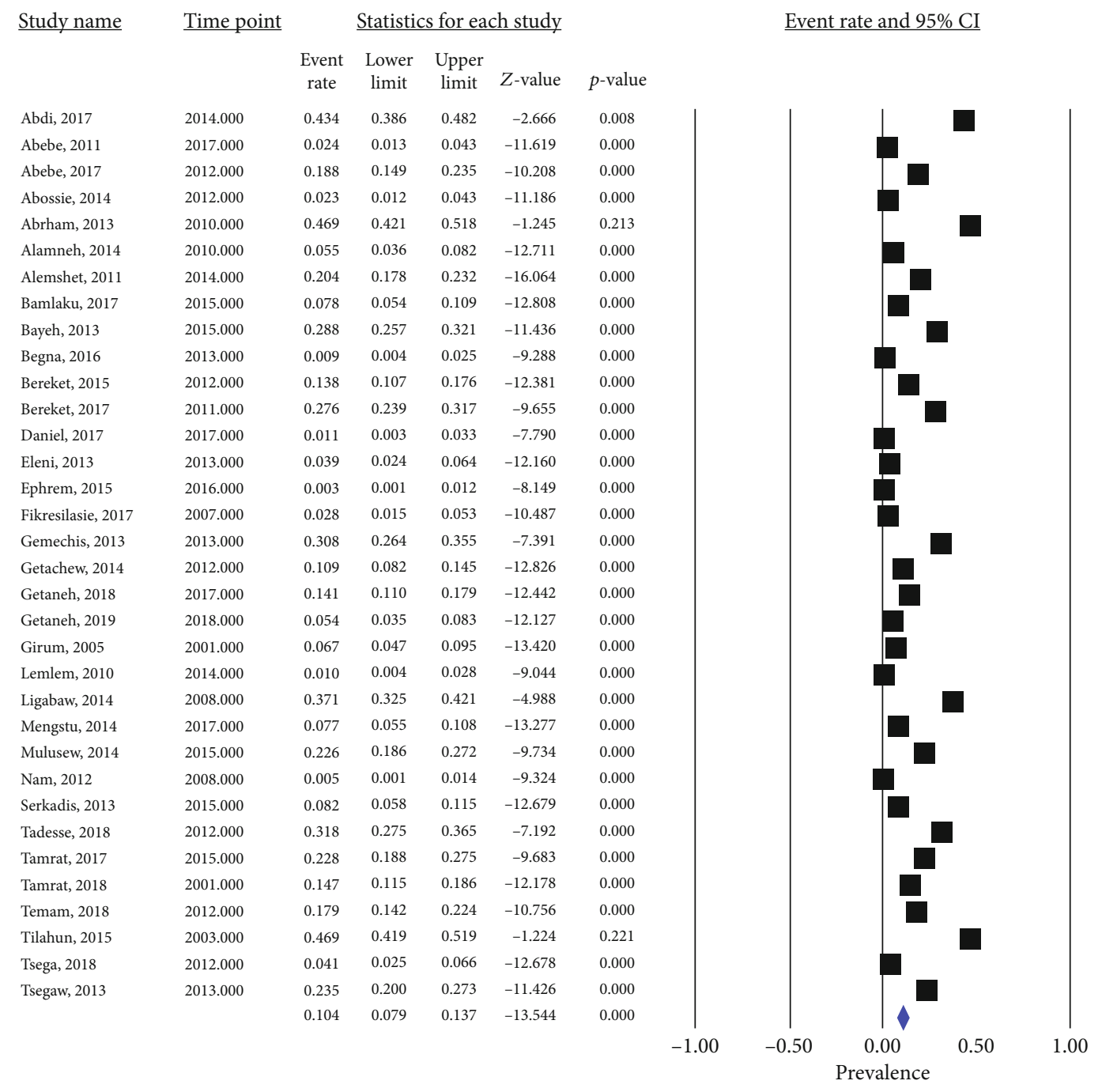

(a)

Figure 5: Continued. 


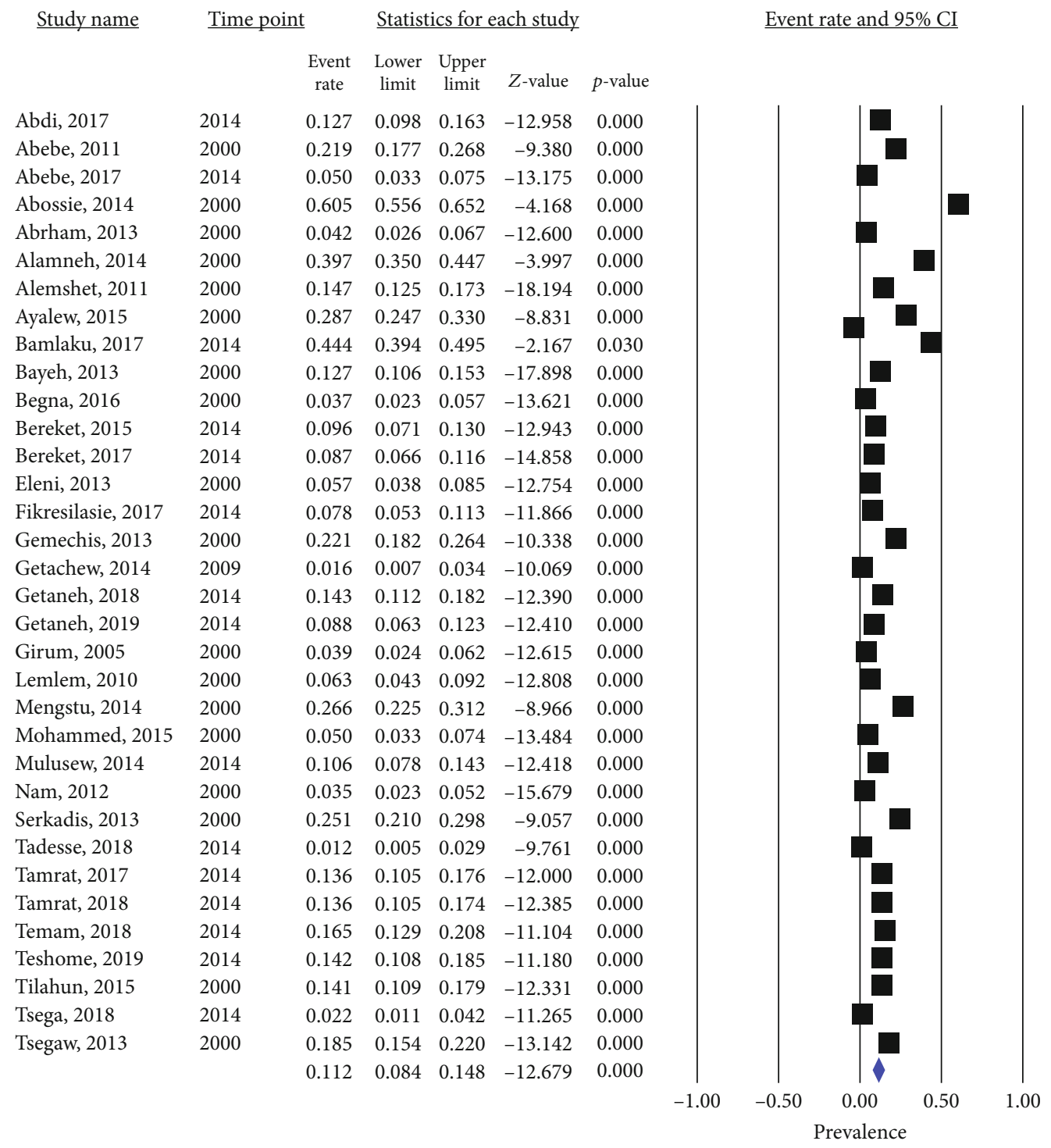

(b)

Figure 5: Continued. 


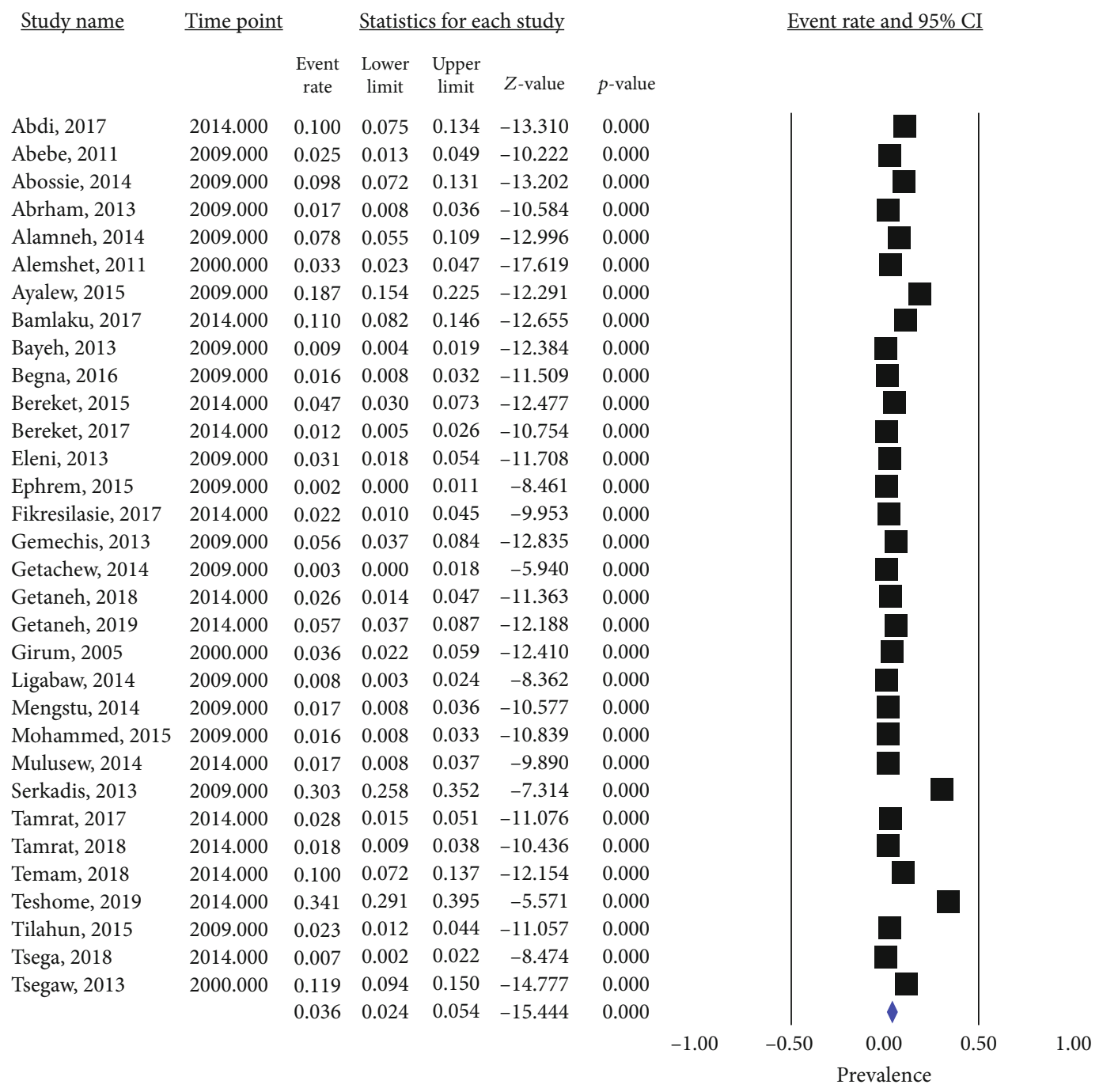

(c)

Figure 5: Forest plot showing pooled prevalence of (a) hookworms, (b) A. lumbricoides, and (c) T. trichiura.

[15-45, 47-52] except 1 which was conducted at the community level [46]. Eleven and nine studies screened stools by formol ether concentration and Kato-Katz techniques, respectively, while seven studies performed both direct wet mount and formol ether concentrations (Table 1).

3.2. Prevalence of Intestinal Parasites. Among a total of 16,266 children participated; 8,200 were infected with at least one IP, yielding a pooled prevalence of $52.0 \%(95 \%$ CI: 44.4-59.5.0; $I^{2}=98.7, p<0.001$ ) (Figure 2). Analysis with stepwise removal of each study revealed a pooled prevalence between 50\% and 53.8\%. Pooled prevalence of IPs among studies reporting both intestinal helminths and protozoa was 53.0\% (95\% CI: 41.0-64.0; $I^{2}=98.4, p<0.001$ ). Similarly, pooled prevalence of IPs among studies reporting only intestinal helminths was 52.0\% (95\% CI: 42.0-62.0; $I^{2}=98.8$, $p<0.001)$. Distribution of studies in the funnel plot, Egger's regression $(p=0.481)$, and Begg's correlation $(p=0.470)$ showed that there was no significant publication bias among the included studies (Figure 3).
3.3. Subgroup Prevalence of Intestinal Parasites. Prevalence of IPs was analyzed by region of study and study period. Region-wise analysis has revealed a pooled prevalence of 60.3\% (95\% CI: 50.1-69.6; $\left.I^{2}=98.3, p<0.001\right)$, 39.7\% (95\% CI: $\left.27.4-53.5 ; I^{2}=98.5, p<0.001\right), 62.1 \%$ (95\% CI: $44.6-$ 76.9; $I^{2}=98.8, p<0.001$ ), and $34.0 \%$ (95\% CI: 11.1-67.9; $I^{2}$ 99.3, $p<0.001)$ in Amhara, Oromia, Southern Nations Nationalities and People's Region (SNNPR), and Tigray, respectively. Subgroup analysis by study period has shown that the pooled prevalence of IPs between 2000 and 2013 was $52.2 \%$ (95\% CI: 41.4-62.7; $I^{2}=98.7, p<0.001$ ) while it was $51.9 \%$ (95\% CI: $41.2-62.4 ; I^{2}=98.4, p<0.001$ ) for studies conducted from 2014 to 2020. Metaregression analysis show that there was no significant trend in decreasing the prevalence of IPs by study period and sample size (Figure 4).

3.4. Prevalence and Trend of Soil-Transmitted Helminths. Thirty-four studies have reported hookworms giving a pooled prevalence of $10.4 \%$ (95\% CI: 7.9-13.7, $I^{2}=97.45, p$ 


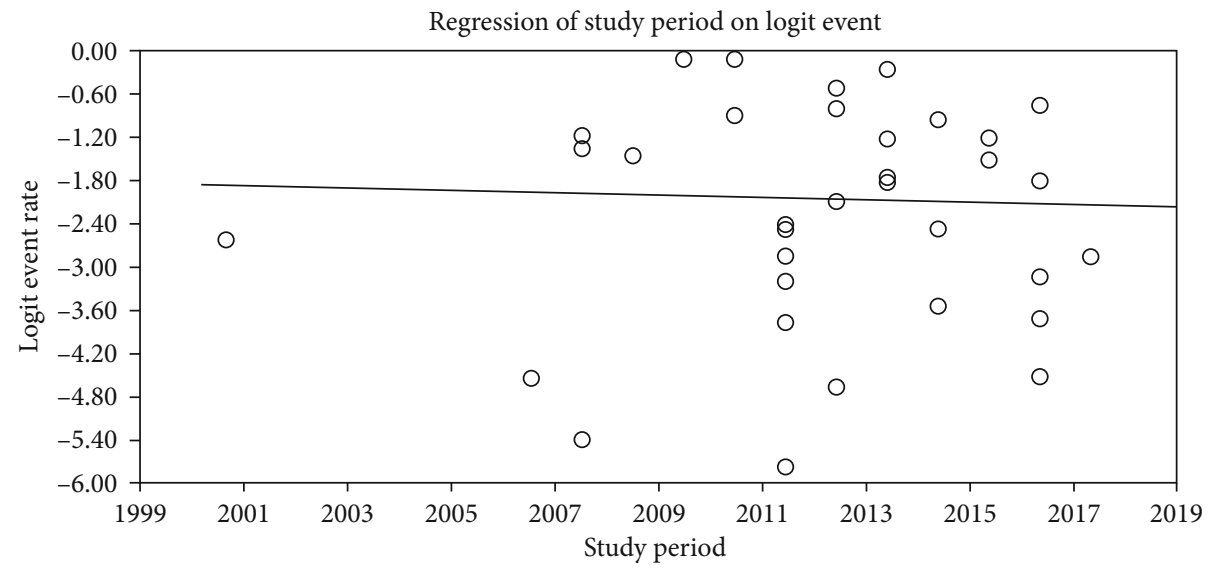

(a)

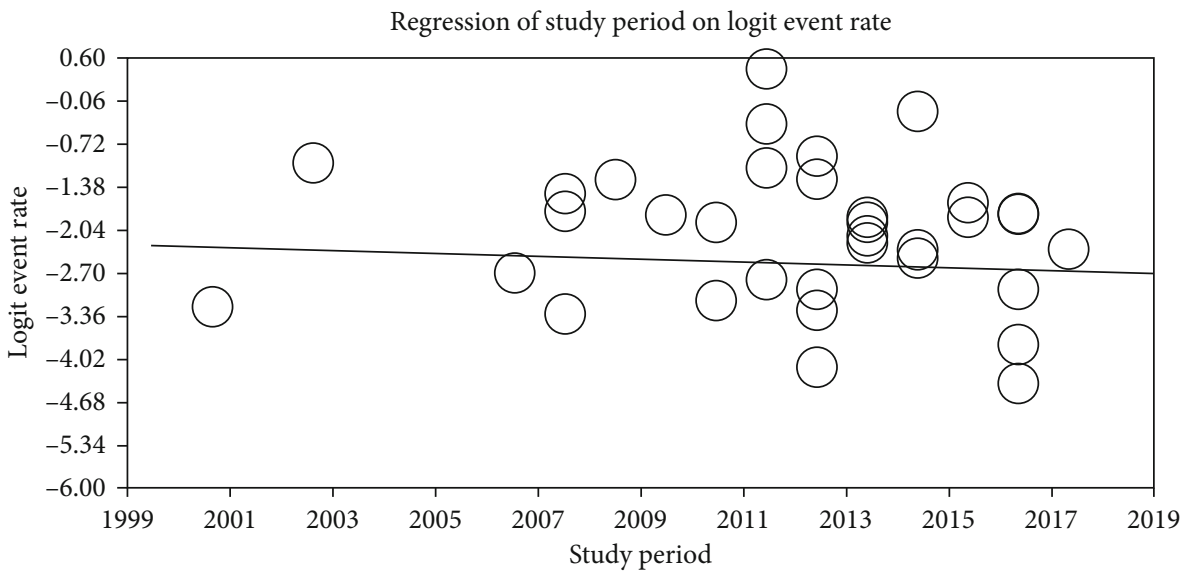

(b)

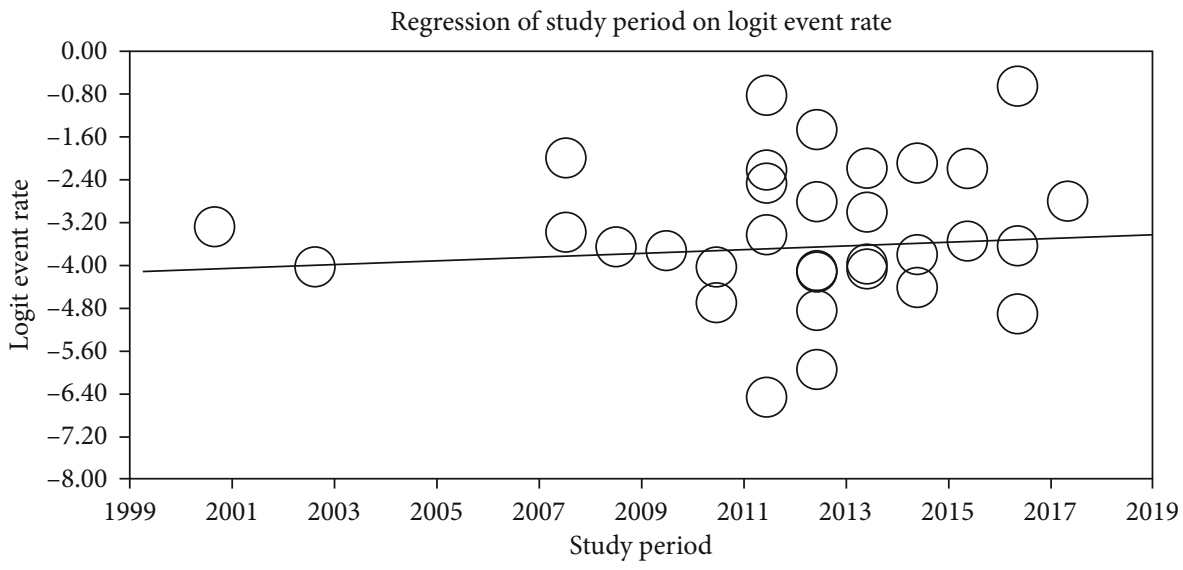

(c)

Figure 6: Metaregression of prevalence of (a) hookworms $(B=-0.01141, p=0.138)$, (b) $A$. lumbricoides $(B=-0.02173, p=0.610)$, and (c) T. trichiura $(B=0.0326, p=0.565)$ by study period.

$<0.001)$. The prevalence of hookworms was $11.7 \%$ (95\% CI: 8.0-16.8) between 2000 and 2013 and 8.7\% (95\% CI: 6.513.4) between the years 2014 and 2020. Analysis of hookworm prevalence has shown a slightly decreasing trend, but it was not statistically significant $(p=0.138)$. The pooled prevalence of $A$. lumbricoides and T. trichiura was $11.2 \%$ (95\% CI: 8.4-14.8, $I^{2}=97.5, p<0.001$ ) and 3.6\% (95\% CI:
2.4-5.4; $\quad I^{2}=96.3, \quad p<0.001$ ), respectively (Table 2; Figures 5 and 6).

\section{Discussion}

The present systematic review and meta-analysis was designed to generate comprehensive data about the national 
prevalence of IPs in general and STHs in particular. Accordingly, studies assessing the prevalence of IPIs, conducted in different regions of Ethiopia, were gathered and analyzed to estimate the national pooled prevalence. The findings provide useful epidemiological data to aid in the control of STHs. The review generated information about the distribution of the big three STHs: A. lumbricoides, hookworm, and T. trichiura in Ethiopia which, in turn, helps to evaluate the success of existing control programs and to plan for the future. It also helps to implement targeted control activities. The overall pooled prevalence of IPs in the present review (52.0\%) was similar to the $47.6 \%$ prevalence in Afghanistan [53] but higher than the prevalence in Iran (38\%) [54], Syria (42.5\%) [55], Turkey (31.8-37.2\%) [1], and Egypt (27\%) [56]. Variations in the distribution of IPs among different geographical settings as well as the type and level of control program implementation across countries might be responsible for these differences.

The pooled prevalence of hookworms in the present review (10.4\%) was in line with review results from South America (11.9\%) [57]. It was lower than the Ethiopian national estimate of $16 \%$ before 10 years [58] and a regionwide survey in the Amhara region (20.6\%) [59] pronounced the impact of MDA and WASH activities since then. Subgroup analysis of the current review also shows the highest prevalence of IPs in the Amhara region. The prevalence was also lower than the pooled prevalence from Nigeria (23.0\%) [54] and Rwanda (31.6\%) [60]. The cumulative number of children included in the review from Nigeria was higher compared to the present review (34,518 vs. 16,266) [54]. Moreover, we have included more recent surveys that the ongoing MDA and WASH programs also impact the prevalence of hookworms unlike in reviews from Rwanda which included studies starting from the year 1940 [60].

Implementation of intervention programs mainly improved healthcare coverage and biannual MDA, and WASH has been thought to bring a decreasing trend of both morbidity and prevalence of STH infections over time. As a result, hookworms show a decreased prevalence between the years 2014 and 2020 (8.7\%) compared to that of the years 2000-2013 (11.7\%). However, the trend in decrement was not significant $(p=0.138)$. This is to the review results from Nepal where hookworm infections significantly decrease between the years 1990 and 2015 [61]. Interventions for STH transmission in Ethiopia primarily target SAC. However, in rural areas where fields are fertilized with night soil, STH species like hookworms may heavily infect adults who, in turn, serve as sources of infection for SAC [59]. Open defecation is common in rural communities of Ethiopia that reinfection of treated children also contributed to the nondecreasing trend of the parasite.

The pooled prevalence was higher than findings from nationwide surveys in Sri Lanka (1.2\%) [62] and Cameroon (1.55\%) [63]. In both countries, the authors used a single Kato-Katz smear, and there is also variation in geographical distribution as well as adoption and level of implementation of control programs across countries.

The pooled prevalence of $A$. lumbricoides in the present review (11.2\%) was in line with findings from Cameroon
(11.48\%) [63]. However, it was lower than results from Nigeria (44.6\%) [64], Rwanda (38.6\%) [60], South America (15.6\%) [57], the Amhara region of Ethiopia (16.8\%) [59], and a previous estimate in Ethiopia which was 37\% [58]. Local studies about mebendazole and albendazole efficacy show that both drugs have more than $95 \%$ efficacy against A. lumbricoides and that the ongoing MDA has substantially decreased the burden of the parasite in Ethiopia [65]. However, the trend of A. lumbricoides was not uniformly decreasing between the years 2000 and $2020(p=0.610)$ with the possible reason of poor WASH implementation in the country [5]. The pooled prevalence of A. lumbricoides in the present review was higher than the review result of $0.75 \%$ from Iran [54] and 2.8\% from Sri Lanka [62].

The pooled prevalence of T. trichiura in the present review (3.6\%) was in line with reviews from the Amhara region (3.8\%) [59] and Sri Lanka which was 4\% [62]. On the other hand, it was lower than review results from South America (12.5\%) [57], Nigeria (31.9\%) [64], Cameroon (18.22\%) [63], Rwanda (27\%) [60], and previous estimates in Ethiopia (30\%) [58]. The pooled prevalence of T. trichiura in the present review was higher than the review result of $0.12 \%$ from Iran [54]. Metaregression analysis by year of study showed that $T$. trichiura has almost constant prevalence between the years 2000 and 2020 in Ethiopia. Both albendazole and mebendazole, drugs used for MDA, have poor efficacy against $T$. trichiura contributing to the nondecreasing trend of parasite prevalence [65].

4.1. Strength and Limitations. The strength of the present review is that it included a large number of studies and has identified the pooled prevalence of the big three STHs which are targeted for control in Ethiopia. As a limitation, the studies included in the present review were conducted in only five regions and we could not get studies from other regions. The primary targets of this review were SAC; however, many studies have included adolescents. Considering a very small proportion of participants are above the age limit of SAC and with the concern not to miss potential findings, we included studies which recruited participants of age 4-25 years old. There was also variation in laboratory techniques used across the reviewed studies. The pooled total IP prevalence in the present study might not show the exact figure in the country because studies not reporting STH were excluded as STHs were the focus of the present review [66].

\section{Conclusion}

The prevalence of IPs in Ethiopia is unacceptably high infecting more than half of the population. This is against the national goal. The federal Ministry of Health has set a goal to eliminate the three STHs (ascariasis, hookworm infection, and trichuriasis) as public health problems by 2025. Despite various control efforts having been made, STHs are with high distribution and their prevalence is not significantly decreasing in Ethiopia. Hence, the integration of the existing control activities (MDA and WASH) accompanied with continuous efforts to create awareness and engagement among the community should be promoted to 
achieve the national goal of STH elimination by 2025 . Alternative treatment options like combination therapy of albendazole/mebendazole with ivermectin should be considered for trichuriasis.

\section{Abbreviations}

IPIs: Intestinal parasitic infections

IPs: Intestinal parasites

MDA: Mass drug administration

SAC: School-age children

STHs: Soil-transmitted helminths

WASH: Water hygiene and sanitation.

\section{Data Availability}

The original data for this study is available from the corresponding author.

\section{Conflicts of Interest}

The authors declare that they have no competing interests.

\section{Authors' Contributions}

GA conceived and designed the study. GA, FM, MN, and $\mathrm{CM}$ selected the studies, extracted the data, and reviewed the manuscript. GA wrote the manuscript. All authors read and approved the final manuscript.

\section{Acknowledgments}

The manuscript entitled "Trend of Soil-Transmitted Helminths in Ethiopian children: A Systematic Review and Meta-Analysis (2000-2018)" is presented as a preprint in the link https://www.researchsquare.com/article/rs-13528/ v1.

\section{Supplementary Materials}

PRISMA checklist. (Supplementary Materials)

\section{References}

[1] P. Okyay, S. Ertug, B. Gultekin, O. Onen, and E. Beser, "Intestinal parasites prevalence and related factors in school children, a western city sample-Turkey," BMC Public Health, vol. 4, no. 1, 2004.

[2] K. Mohammed, M. Abdullah, and J. Omar, "Intestinal parasitic infection and assessment of risk factors in north-western Nigeria: a community based study," International Journal of Pharma Medicine and Biological Sciences, vol. 4, no. 2, pp. 141-145, 2015.

[3] L. R. Pullan and S. J. Brooker, "The global limits and population at risk of soil-transmitted helminth infections in 2010," Parasites \& Vectors, vol. 5, no. 1, 2012.

[4] Ethiopian Federal Ministry of Health: Second edition of national neglected tropical diseases master plan, 2016.

[5] Federal Democratic Republic Of Ethiopia. Demographic and health survey, Addis Ababa, Central statistical Agency, 2016.
[6] B. Habtamu and H. Kloos, The epidemiology and ecology of health and diseases in Ethiopia In: Intestinal parasitism, Shama Books,, Addis Ababa, Ethiopia, 2006.

[7] L. Jemaneh, "Comparative prevalences of some common intestinal helminth infections in different altitudinal regions in Ethiopia," Ethiopian Medical Journal, vol. 36, pp. 1-8, 1998.

[8] M. O. Harhay, J. Horton, and P. L. Olliaro, "Epidemiology and control of human gastrointestinal parasites in children," Expert Review of Anti-Infective Therapy, vol. 8, no. 2, pp. 219-234, 2010.

[9] R. K. Chandra, "Nutrition, immunity and infection: From basic knowledge of dietary manipulation of immune responses to practical application of ameliorating suffering and improving survival," Proceedings of the National Academy of Sciences of the United States of America, vol. 93, no. 25, pp. 1430414307, 1996.

[10] W. Wakabi, "Extension workers drive Ethiopia's primary health care," The Lancet, vol. 372, no. 9642, p. 880, 2008.

[11] N. Nebiyu, M. Birhan, K. Biruck, D. Kebede, E. Ephrem, T. Gemechu et al., "Ethiopia schistosomiasis and soiltransmitted helminthes control programme: progress and prospects," Ethiopian Medical Journal, vol. 55, no. 1, pp. 7580, 2017.

[12] “Geography of Ethiopia," 2019, https://en.wikipedia.org/wiki/ Geography_of_Ethiopia.

[13] "Ethiopia-World population review," 2019, http:// worldpopulationreview.com/countries/ethiopia-population/.

[14] D. LA Moher, A. Liberati, J. Tetzlaff, D. G. Altman, and The PRISMA Group, "Preferred reporting items for systematic reviews and meta-analyses: the PRISMA statement," PLoS Medicine, vol. 6, no. 7, 2009.

[15] D. G. Feleke, S. Arega, M. Tekleweini, K. Kindie, and A. Gedefie, "Schistosoma mansoni and other helminthes infections at Haike primary school children, North-East, Ethiopia: a cross-sectional study," BMC Research Notes, vol. 10, no. 1, p. 609, 2017.

[16] Y. Alemeshet, M. Yoseph, and K. Seleshi, "Prevalence and predictors of intestinal helminthiasis among school children in Jimma zone; a cross-sectional study," Ethiopian Journal of Health Sciences, vol. 21, no. 3, pp. 167-174, 2011.

[17] N. Nam Linh, G. Bizu, A. Nigusu, K. Abera, A. Michelle, and B. Yemane, "Intestinal parasitic infection and nutritional status among school children in Angolela, Ethiopia," Journal of Preventive Medicine And Hygiene, vol. 53, no. 3, pp. 157-164, 2012.

[18] B. Alemayehu and Z. Tomass, “_Schistosoma mansoni_infection prevalence and associated risk factors among schoolchildren in Demba Girara, Damot Woide District of Wolaita Zone, Southern Ethiopia," Asian Pacific Journal of Tropical Medicine, vol. 8, no. 6, pp. 457-463, 2015.

[19] M. Abdi, E. Nibret, and A. Munshea, "Prevalence of intestinal helminthic infections and malnutrition among schoolchildren of the Zegie peninsula, northwestern Ethiopia," Journal of Infection and Public Health, vol. 10, no. 1, pp. 84-92, 2017.

[20] A. Alemu, A. Atnafu, Z. Addis et al., "Soil transmitted helminths and Schistosoma mansoni infections among school children in Zarima town, northwest Ethiopia," BMC Infectious Diseases, vol. 11, no. 1, p. 189, 2011.

[21] D. Abraham and E. Berhanu, "Association between intestinal helminth infections and underweight among school children in Tikur Wuha elementary school, northwestern Ethiopia," 
Journal of Infection and Public Health, vol. 6, pp. 125-133, 2013.

[22] A. Abera and E. Nibret, "Prevalence of gastrointestinal helminthic infections and associated risk factors among schoolchildren in Tilili town, Northwest Ethiopia," Asian Pacific Journal of Tropical Medicine, vol. 7, no. 7, pp. 525-530, 2014.

[23] B. Tadege and T. Shimelis, "Infections with Schistosoma mansoni and geohelminths among school children dwelling along the shore of the Lake Hawassa, southern Ethiopia," PLoS One, vol. 12, no. 7, 2017.

[24] B. Abera, G. Alem, M. Yimer, and Z. Herrador, "Epidemiology of soil-transmitted helminths, Schistosoma mansoni, and haematocrit values among schoolchildren in Ethiopia," The Journal of Infection in Developing Countries, vol. 7, no. 3, pp. 253-260, 2013.

[25] B. Alemayehu, Z. Tomass, F. Wadilo, D. Leja, S. Liang, and B. Erko, "Epidemiology of intestinal helminthiasis among school children with emphasis on Schistosoma mansoni infection in Wolaita zone, Southern Ethiopia," BMC Public Health, vol. 17, no. 1, p. 587, 2017.

[26] E. Tefera, J. Mohammed, and H. Mitiku, "Intestinal helminthic infections among elementary students of Babile town, eastern Ethiopia," Pan African Medical Journal, vol. 20, p. 50, 2015.

[27] F. Samuel, A. Demsew, Y. Alem, and Y. Hailesilassie, "Soil transmitted helminthiasis and associated risk factors among elementary school children in Ambo town, western Ethiopia," BMC Public Health, vol. 17, no. 1, p. 791, 2017.

[28] G. Gemechis, M. Sissay, and G. Yitibarek, "Soil-transmitted helminth infections and their associations with hemoglobin concentration and anthropometric measurements of school children in Jimma Arjo primary school children, Oromiya region, western Ethiopia," International Journal of Innovation in Science and Mathematics, vol. 1, no. 1, pp. 2347-9051, 2013.

[29] G. Alebie, B. Erko, M. Aemero, and B. Petros, "Epidemiological study on Schistosoma mansoni infection in Sanja area, Amhara region, Ethiopia," Parasites Vectors, vol. 7, no. 1, p. 15, 2014.

[30] T. Girum, "The prevalence of intestinal helminthic infections and associated risk factors among school children in Babile town, eastern Ethiopia," Ethiopian Journal of Health Development, vol. 19, no. 2, pp. 140-147, 2005.

[31] L. Worku, D. Damte, M. Endris, H. Tesfa, and M. Aemero, "Schistosoma mansoni infection and associated determinant factors among school children in Sanja town, Northwest Ethiopia," Journal of Parasitology Research, vol. 2014, 7 pages, 2014.

[32] A. Megbaru, H. Asrat, and B. Gessessew, "Prevalence of intestinal schistosomiasis and soil transmitted helminthiasis among primary school children in Umolante district, South Ethiopia," Clinical Medicine Research, vol. 3, no. 6, pp. 174-180, 2014.

[33] W. Mengistu, W. Melaku, and F. Tesfu, "The prevalence of intestinal helminthic infections and associated risk factors among school children in Lumame town, Northwest Ethiopia," Journal of Parasitology and Vector Biology, vol. 6, no. 10, pp. 156-165, 2014.

[34] S. Mohammed, D. Tadesse, and T. Zewdneh, "Prevalence of intestinal helminths and associated risk factors in rural school-children in Were-Abaye Sub District Tigray region, Northern Ethiopia," Acta Parasitologica Globalis, vol. 6, no. 1, pp. 29-35, 2015.

[35] S. Debalke, A. Worku, N. Jahur, and Z. Mekonnen, "Soil transmitted helminths and associated factors among schoolchildren in government and private primary school in Jimma town, Southwest Ethiopia," Ethiopian Journal of Health Sciences, vol. 23, no. 3, pp. 237-244, 2013.

[36] T. Hailegebriel, "Prevalence of intestinal parasitic infections and associated risk factors among students at Dona Berber primary school, Bahir Dar, Ethiopia," BMC Infectious Diseases, vol. 17, no. 1, p. 362, 2017.

[37] T. Ibrahim, E. Zemene, Y. Asres et al., "Epidemiology of soiltransmitted helminths and Schistosoma mansoni: a base-line survey among school children, Ejaji, Ethiopia," The Journal of Infection in Developing Countries, vol. 12, no. 12, pp. 1134-1141, 2018.

[38] T. Bekana, W. Hu, S. Liang, and B. Erko, "Transmission of Schistosoma mansoni in Yachi areas, southwestern Ethiopia: new foci," Infectious Diseases of Poverty, vol. 8, no. 1, p. 1, 2019.

[39] T. Alelign, A. Degarege, and B. Erko, "Soil-transmitted helminth infections and associated risk factors among schoolchildren in Durbete town, northwestern Ethiopia," Journal of Parasitology Research, vol. 2015, Article ID 641602, 5 pages, 2015.

[40] T. Teshale, S. Belay, D. Tadesse, A. Awala, and G. Teklay, "Prevalence of intestinal helminths and associated factors among school children of Medebay Zana wereda; north western Tigray, Ethiopia 2017," BMC Res Notes, vol. 11, no. 1, p. 444, 2018.

[41] W. Abebe, "Prevalence of Helicobacter pylori and intestinal parasite and their associated risk factors among school children at Selam Fire Elementary School in Akaki Kality, Addis Ababa, Ethiopia," 2017, http://etd.aau.edu.et/bitstream/ handle/123456789/3011/Abebe\%20Worku.pdf? sequence $=$ 1\&isAllowed $=\mathrm{y}$.

[42] A. Abossie and M. Seid, "Assessment of the prevalence of intestinal parasitosis and associated risk factors among primary school children in Chencha town, southern Ethiopia," BMC Public Health, vol. 14, no. 1, p. 166, 2014.

[43] A. Jejaw, E. Zemene, Y. Alemu, and Z. Mengistie, "High prevalence of Schistosoma mansoni and other intestinal parasites among elementary school children in Southwest Ethiopia: a cross-sectional study," BMC Public Health, vol. 15, no. 1, p. 600, 2015.

[44] T. Begna, T. Solomon, Z. Yohannes, and A. Eden, "Intestinal parasitic infections and nutritional status among primary school children in Delo-Mena district, South eastern Ethiopia," Iranian Journal of Parasitology, vol. 11, no. 4, pp. 549558, 2016.

[45] E. Kidane, S. Menkir, A. Kebede, and M. Desta, "Prevalence of intestinal parasitic infections and their associations with anthropometric measurements of school children in selected primary schools, Wukro town, eastern Tigray, Ethiopia," Scientific Journal of Zoology, vol. 2, no. 12, pp. 117-132, 2013.

[46] G. Alemu, Z. Aschalew, and E. Zerihun, "Burden of intestinal helminths and associated factors three years after initiation of mass drug administration in Arbaminch Zuria district, Southern Ethiopia," BMC Infectious Diseases, vol. 18, no. 1, p. 435, 2018.

[47] G. Alemu, A. Abossie, and Z. Yohannes, "Current status of intestinal parasitic infections and associated factors among primary school children in Birbir town, Southern Ethiopia," BMC Infectious Diseases., vol. 19, no. 1, p. 270, 2019.

[48] L. Lemlem, E. Berhanu, and H. Asrat, "Current status of intestinal schistosomiasis and soiltransmitted helminthiasis among primary school children in Adwa town, Northern Ethiopia," 
Ethiopian Journal of Health Development, vol. 24, no. 3, pp. 191-197, 2010.

[49] A. A. Mulusew, "Parasitic infection and associated factors among the primary school children in Motta town, western Amhara, Ethiopia," American Journal of Public Health Research, vol. 2, no. 6, pp. 248-254, 2014.

[50] T. Hailu, M. Alemu, B. Abera et al., "Multivariate analysis of factors associated with Schistosoma mansoni and hookworm infection among primary school children in rural Bahir Dar, Northwest Ethiopia," Tropical Diseases, Travel Medicine and Vaccines, vol. 4, no. 1, 2018.

[51] H. Tamirat, "Undernutrition, intestinal parasitic infection and associated risk factors among selected primary school children in Bahir Dar, Ethiopia," BMC Infectious Diseases, vol. 18, no. 1, p. 394, 2018.

[52] T. Fentie, S. Erqou, M. Gedefaw, and A. Desta, "Epidemiology of human fascioliasis and intestinal parasitosis among schoolchildren in Lake Tana Basin, Northwest Ethiopia," Transactions of the Royal Society of Tropical Medicine and Hygiene, vol. 107, no. 8, pp. 480-486, 2013.

[53] A. Gabrielli, M. Ramsan, C. Naumann et al., "Soil-transmitted helminths and haemoglobin status among Afghan children in World Food Programme assisted schools," Journal of Helminthology, vol. 79, no. 4, pp. 381-384, 2005.

[54] A. Daryani, S. Hosseini-Teshnizi, S. A. Hosseini et al., "Intestinal parasitic infections in Iranian preschool and school children: a systematic review and meta-analysis," Acta Tropica, vol. 169, pp. 69-83, 2017.

[55] A. Al-kafri and A. Harba, "Intestinal parasites in basic education pupils in urban and rural Idlb," Journal of Laboratory Diagnosis, vol. 5, pp. 2-7, 2009.

[56] F. A. El-Soud, R. A. Salama, and N. S. Taha, "Predictors of the intestinal parasitic infection among preschool children in rural lower, Egypt," Egyptian Journal of Community Medicine, vol. 27, pp. 17-34, 2009.

[57] F. Chammartin, R. G. C. Scholte, L. H. Guimarães, M. Tanner, J. Utzinger, and P. Vounatsou, "Soil-transmitted helminth infection in South America: a systematic review and geostatistical meta-analysis," The Lancet Infectious Diseases, vol. 13, no. 6, pp. 507-518, 2013.

[58] Z. Tadesse, A. Hailemariam, and J. H. Kolaczinski, "Potential for integrated control of neglected tropical diseases in Ethiopia," Transactions of the Royal Society of Tropical Medicine and Hygiene, vol. 102, no. 3, pp. 213-214, 2008.

[59] S. T. Fikresilasie, "Status of soil-transmitted Helminths infection in Ethiopia," American Journal of Health Research, vol. 3, no. 3, pp. 170-176, 2015.

[60] N. Rujeni, D. Morona, E. Ruberanziza, and H. D. Mazigo, "Schistosomiasis and soil-transmitted helminthiasis in Rwanda: an update on their epidemiology and control," Infectious Diseases of Poverty, vol. 6, no. 1, p. 8, 2017.

[61] R. Kunwar, L. Acharya, and S. Karki, "Trends in prevalence of soil-transmitted helminth and major intestinal protozoan infections among school-aged children in Nepal," Tropical Medicine and International Health, vol. 21, no. 6, pp. 703719, 2016.

[62] N. R. de Silva, "The soil-transmitted helminths in Sri Lanka: a review of the recent literature," Sri Lankan Journal of Infectious Diseases, vol. 8, no. 2, pp. 60-68, 2018.

[63] L. A. Tchuem Tchuenté, R. I. Kamwa Ngassam, L. Sumo et al., "Mapping of schistosomiasis and soil-transmitted helminthia- sis in the regions of centre, east and west Cameroon," PLoS Neglected Tropical Diseases, vol. 6, no. 3, article e1553, 2012.

[64] N. K. Solomon, "Prevalence and distribution of soiltransmitted helminth infections in Nigerian children: a systematic review and meta-analysis," Infectious Diseases of Poverty, vol. 7, no. 1, p. 69, 2018.

[65] E. Tefera, T. Belay, S. K. Mekonnen, A. Zeynudin, and T. Belachew, "Therapeutic efficacy of different brands of albendazole against soil transmitted helminths among students of Mendera elementary school, Jimma, southwest Ethiopia," Pan African Medical Journal, vol. 22, p. 252, 2015.

[66] A. Getaneh, M. Feleke, N. Mezgebu, and M. Chalachew, Trend of Soil Transmitted Helminths in Ethiopian Children: A Systematic Review and Meta-Analysis (2000-2018), Research square, 2020. 